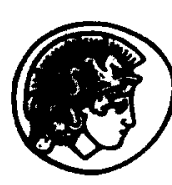

PERGAMON Computers \& Industrial Engineering 37 (1999) 355-358

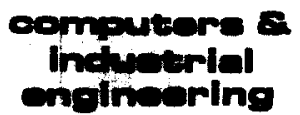

\title{
AN AUTOMATIC OPTICAL SENSOR FOR VESSELS AND FIBBERS QUALITY INSPECTION IN PULP PRODUCTION
}

\author{
P. Carvalho ${ }^{\dagger}$, H. Araúioł, A. Dourado ${ }^{\dagger}$ \\ † CISUC, Dep. de Eng. Informática da Universidade de Coimbra \\ $\ddagger$ Dep. de Eng. Electrotécnica de Universidade de Coimbra \\ Pólo ll, P3030 Coimbra, Portugal
}

Abstract: Wood quality assessment is an important issue in the pulp and paper industry. In this paper an automatic visual inspection system for wood structural quality measurement is described. To improve statistical image segmentation a new logarithm based global contrast enhancement technique is described. Region identification and reconstruction is performed based on local morphological operators with constrained merge operations. A measurement technique, build-up upon a constrained optimisation problem, is described for fibber wall width computation. The method proves computational efficiency. The described inspection system uses only two control parameters. (C) 1999 Elsevier Science Ltd. All rights reserved.

Keywords: Quality Control; Production Systems; Automatic Visual Inspection; Vision and Image Processing; Contrast Enhancement; Microscope Image Analyses; Pulp and Paper

\section{INTRODUCTION}

Several mechanical properties of paper are highly dependent on the microscopic characteristics of the applied wood in its production. To obtain the best raw materials possible for pulp production, several genetic studies are permanently conducted to improve the applied wood species. This leads to a considerable amount of samples to be processed. Each sample is manually analysed for its structural properties with respect to (i) the fibbers' (Lumen diameters, fibber wall width and fibber density), or with respect to (ii) the vessels' (tangential diameters and vessels' density) distribution. Given, however, the large number of samples, this task tends to be highly timed consuming. Another disadvantage of this quality inspection approach is the substantial accuracy decrease, during the inspection process, due to the fatigue of the human operator. These facts are stimulating the development of computer vision based inspection systems in an attempt to increase the inspection performance and to improve data consistency. The major obstacle in computer vision based wood structure inspection arises due to valid region identification for measurement. For accuracy reasons, it is imperative that the intended regions (vessels and fibbers) are computed with the least possible size and shape distortion. The images obtained from microscope observations of wood samples usually exhibit a global lack of contrast, which is a major constraint for region segmentation. On the other hand, it often happens that during the sample preparation several obstructions occur due to organic tissue depositions, as well as wall ruptures between adjacent vessels, which in turn lead to fusion of physically distinct regions.

Recent developments in artificial intelligence have lead to the introduction of powerful strategies for image segmentation based on fuzzy sets, neural networks and genetic algorithms (Udupta and Samarasekera, 1996). Other non-statistical strategies explore geometrical properties of the image surface. These methods, generally, use mathematical morphology to extract the constituent parts from the image background. Although these recent developments have strongly improved the performance of the segmentation stage in computer vision systems, they are all very computationally demanding. Therefore, it is seen that traditional segmentation strategies based on statistical image analysis are still to be considered and improved for practical computer implementations, as is done in this paper for wood structure quality inspection.

The solution described involves the application of a three stage "segmentation algorithm" where some a priori known morphological properties are used. In a first stage the effect of low contrast in the image is attenuated. For this purpose a new global contrast enhancement algorithm is introduced. This method is described in section 2. 0360-8352/99 - see front matter $\odot 1999$ Elsevier Science Ltd. All rights reserved.

PII: S0360-8352(99)00092-3 


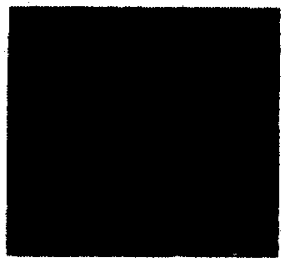

(a)

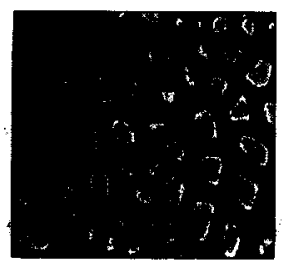

(b)

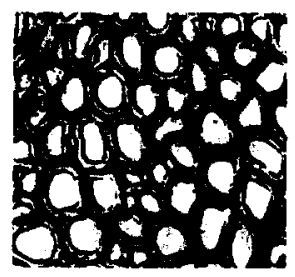

(c)

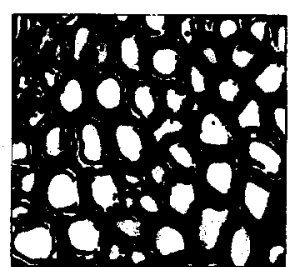

(d)

Fig. 1: a) Input image; b) Contrast enhanced image; c) Threshold result of a); d) Threshold result of b).

After contrast enhancement a threshold is applied to the image using a minimum cross-entropy valley searching method, which leads to a first estimation of the intended regions. This operation is followed by an identification and a reconstruction strategy for vessels and fibbers using conditional and local morphological operators, and constrained merge operations. This procedure will be outlined in section 3 . After the segmentation phase, the system proceeds with a measurement algorithm where all fundamental parameters for wood quality assessment are obtained. For this purpose a new technique based on a constrained optimisation problem is introduced. This operation proves computational efficiency and it will be described in section 4 . Finally, in section 5 the main conclusions are presented.

\section{CONTRAST ENHANCEMENT}

Global low contrast is a common characteristic to wood samples' images. These images usually exhibit histograms with high-density populations in narrow bands of the gray-scale. This behaviour leads to inadequate unimodal or bimodal with subtle valley histogram formations, which is a major constraint for statistical threshold computation. The internal regions of vessels and fibbers are characterised by relative homogenous and constant gray level distributions, which correspond to the upper bound of the gray-scale. These regions occupy, in average, $50 \%$ of the image's area. Therefore, to enhance the contrast between these regions and the surrounding background the following mapping function is introduced $(f(x, y)$ - gray level of pixel at co-ordinates $(x, y) ; L$ - maximum gray level):

$$
\begin{gathered}
\forall x=\{1, \ldots, P\}, y=\{1, \ldots Q\}: f(x, y)=\left\{\begin{array}{l}
f^{\prime}(x, y) \Leftarrow f^{\prime}(x, y) \leq L \\
L \Leftarrow f^{\prime}(x, y)>L
\end{array}\right. \\
f^{\prime}(x, y)=\frac{\left[f(x, y) \frac{\ln (f(x, y)+0.1)}{\ln (\operatorname{Max}(f(x, y))+0.1)}\right]^{2}}{\mu} \alpha, \quad \alpha=\left\{\begin{array}{l}
1-\beta \Leftarrow f(x, y)<\mu \\
1+\beta \Leftarrow f(x, y) \geq \mu
\end{array}\right.
\end{gathered}
$$

The function described in equations 1 and 2 is similar to the logarithmic function. However, its shape is controllable through parameters $\beta$ (we use $\beta=0.1$ ) and $\mu$. The transfer curve's slope is adjusted by $\beta$, while parameter $\mu$ serves as a threshold between attenuation and amplification. Since, in average, the intended regions occupy $50 \%$ of the image, this value is taken as the average gray level of the histogram. The contrast enhancement properties of this operator tends to split the pixels' population into two sub-populations, implying, in practical terms, the formation of a valley in unimodal histograms or its accentuation in bimodal ones. Another useful consequence of applying this method is that potential difficulties in post-processing, arising from the obstructions with organic tissue inside the vessels, are partially solved, since the local contrast will be decreased in those areas. In Fig. 1b some results of this mapping function can be observed.

\section{REGION IDENTIFICATION AND RECONSTRUCTION}

After contrast enhancement the image is segmented using a minimum cross-entropy threshold searching method (Brink and Pendock, 1996), which leads to a first estimation of the intended regions. However, it is seen that the obtained binary image exhibits several noisy regions, which may appear isolated as well as connected to the intended ones. It is also observed that, generally, several vessels' fusion occur, since wall ruptures between neighbour vessels usually exhibit similar gray level distributions as the intended regions, i.e., the vessels. To correct for these anomalies, an erosion operation (equation 3 ) is applied using a symmetrical $3 \times 3$ 4-connected structuring element $B$. This operator is applied globally to the image $n$ times $\left(F_{i}^{k}\right.$ - image after $i$ erosion operations; $F_{0}^{b}=$ binary image). 


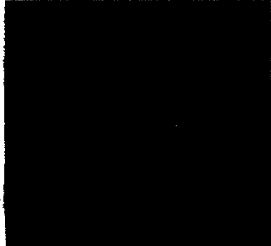

(a)

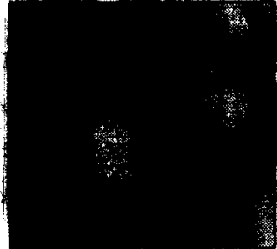

(b)

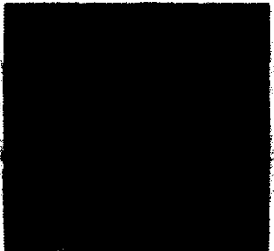

(c)

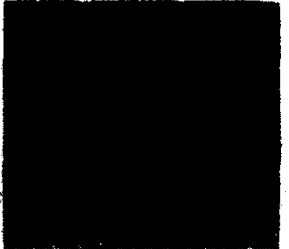

(d)

Fig. 2: Identification results: (a) and (b) Vessels identification; (c) and (d) Fibbers identification.

$$
F_{i}^{E} \leftarrow \bigcap_{x \in B}\left(F_{i-1}^{E}+x\right), \quad i=\{1, \ldots, n\}
$$

It is seen that, due to irregular shapes, it may happen that region separation occur. Region splitting is identified by an inclusion test between the erosion result and the original binary image as described in (Carvalho et. al., 1997, 1998). Separations of regions in vessels' analysis may correspond to two distinct situations: (i) splitting due to the existence of inner holes and (ii) splitting due to the separation of fused vessels. It is considered that two regions $R_{j 1}^{E}$ and $R_{j 2}^{E}$, with centroids $p_{j 1}$ and $p_{j 2}$ respectively, belong to the same vessel if the following constrain are validated:

1. Regions may not have been split due the existance of inner holes $\left(R^{\text {Cont }}\right)$. This is verified by counting the number of eroded pixels between the pair of regions under analysis. Theortically it should be equal to $2 n$. However, to account for less regular shapes we use $\zeta$ (we use $\zeta=2.5$ ). That is $(F$-image):

$$
\#\left\{\overline{p_{j 1} p_{j 2}} \cap\left\{F \backslash\left\{R_{j 1}^{E}, R_{j 2}^{E}, R^{\text {Cont }}\right\}\right\}\right\} \leq \zeta n
$$

2. Regions that belong to distinct neighbour vessels should have similar sizes. This is verified by equation (5) ( $T_{\text {area }}$ - user defined parameter; $C_{k}$ - number of regions which belonged to the same structure):

$$
\frac{\operatorname{Min}_{R_{j}^{E} \in C_{k}}\left\{\operatorname{Area}\left(R_{j}^{E}\right)\right)\left(\# C_{k}-1\right)}{\left[\sum_{R_{j}^{E} \in C_{k}} \operatorname{Area}\left(R_{j}^{E}\right)\right]-\operatorname{Min}_{R_{j}^{E} \in C_{k}}\left\{\operatorname{Area}\left(R_{j}^{E}\right)\right\}} \geq T_{\text {area }}
$$

After the set of regions of each element in the image has been identified, the original region is reconstructed using a conditional dilation operator inside a dilation window $W$ as explained in (Carvalho et. al., 1998) (note that $\left.F_{0}^{R}=F_{n}^{E}\right)$ :

$$
F_{i}^{R} \leftarrow\left\{\bigcup_{x \in B}\left(F_{i-1}^{R}+x\right)\right\} \cap F^{B} \cap W, \quad i=\{1, \ldots, n\}
$$

In Fig. 2 several identification results of vessels and fibbers may be observed.

\section{MEASURMENT USING AN ADJACENCY MATRIX}

Measurement of fibber properties involves the computation of two distinct types of data: (i) the Lumen diameters, which are quite straightforward to determine, and (ii) the width of fibber wall. The later is computed from an adjacency matrix $T_{N R \times N R}=\left\lfloor d_{i j}\right\rfloor$ (NR - number of identified regions) which is obtained by solving the following constrained optimisation problem: let $d_{i j}$ be the modulus of the line segment $\bar{d}_{i j}$ defined by points $p_{i}$ and $p_{j}$, which correspond to the centroids of regions $R_{i}$ and $R_{j}$ respectively, $i, j \in\{1, \ldots, N R\}$, then:

Criterion:

$$
J=\operatorname{Max}\left\{\sum_{i=1}^{N R} \sum_{j=1, j \neq 1}^{N R} \Xi(T(i, j))\right\}, \quad \Xi(d)=\left\{\begin{array}{l}
1 \Leftarrow d \neq 0 \\
0 \Leftarrow d=0
\end{array}\right.
$$

Constraints: (i) $\forall k \in\{1, \ldots, N R\} \backslash\{i, j\}: R_{k} \cap \bar{d}_{i j}=\varnothing ;$ (ii) $\forall \bar{d}_{k z}, k, z \in\{1, \ldots, N R\} \backslash\{i, j\}: d_{k z}<d_{i j} \wedge \bar{d}_{k z} \cap \bar{d}_{i j}=\varnothing$.

Constraint (1) can be interpreted as: if the line segment $\bar{d}_{i j}$ crosses a region, this means that there exists another region between $R_{i}$ and $R_{j}$ in the image, and, therefore, $R_{i}$ and $R_{j}$ are not adjacent to each other. As for constraint (2), it is seen that if $d_{i j}$ and $\bar{d}_{k z}$ cross, then only one of them correspond to an adjacency indication, since we are only interested in wall widths and not in their lengths. In these situations only the smallest line segment is retained. 
These constraints are implemented by (it should be noted that matrix $T$ is simetrical, and, therefore, only half of the matrix has to be analysed): (i) if $T(i, j) \neq 0 \wedge \exists k \in\{1, \ldots, N R\} \backslash\{i, j\}: R_{k} \cap d_{i j} \neq \varnothing$, then eliminate adjacency indication $(T(i, j)=0)$; (ii) if $\exists \bar{d}_{k z}, k, z \in\{1, \ldots, N R\} \backslash\{i, j\}: T(i, j) \neq 0 \wedge T(k, z) \neq 0 \wedge d_{k z}<d_{i j} \wedge d_{k z} \cap d_{i j}=\varnothing$ then eliminate adjacency indication $\left(T(i, j)=0\right.$ ). If $R_{k} \cap d_{i j} \neq \varnothing$ then it is seen that there exists at least one point of $R_{k}$ 's contour which belongs to $d_{i j}$. This is easly determined by the following equation ( $e_{i}$ - chain direction at point $p_{i}$; $\rho=x \cos \theta+y \sin \theta-$ polar equation of $d_{i j} ; N_{\text {Coni }}-$ number of contour points of $R_{k}$ ):

\begin{tabular}{ccccccccc}
\multicolumn{10}{c}{$\exists$} & $z \in\left\{1, \ldots, N_{\text {Cont }}\right\}:\left\|\Delta \rho_{z}\right\| \leq \sqrt{0.5}$ \\
& $\Delta \rho_{i}=\Delta \rho_{i-1}+\Gamma\left(e_{i}\right), \Delta \rho_{0}=0$ \\
\hline Chain $\operatorname{code}$ & 0 & 1 & 2 & 3 & 4 & 5 & 6 & 7 \\
\hline$\Gamma$ & $-\cos \theta$ & $\sin \theta-\cos \theta$ & $\sin \theta$ & $\sin \theta+\cos \theta$ & $\cos \theta$ & $\cos \theta-\sin \theta$ & $-\sin \theta$ & $-\cos \theta-\sin \theta$ \\
\hline \multicolumn{8}{c}{ Table 1: Definition of function $\Gamma}$.
\end{tabular}

It is often observed that, after reconstruction, small regions appear in the image, whose origin may be as due to small fibbers, as due to fibber walls', that remained in $F$. To avoid erroneous adjacency indications induced by these regions, they are identified and adjacency indications related to them are eliminated: let $R_{1}$ and $R_{2}$ be two circular regions of radius $r_{1}$ and $r_{2}$ (let $r_{1}>r_{2}$ ), respectively. The values of $r_{1}$ and $r_{2}$ will be increased during dilation by $n$ pixels. The relation between the circles' areas, before and after dilation, is given by:

$$
\Phi_{r}=\frac{A_{r+n}}{A_{r}}=1+\frac{2 n}{r}+\left(\frac{n}{r}\right)^{2} \Rightarrow 1>\Phi_{r_{2}}>\Phi_{r_{1}}
$$

That is, smaller regions suffer higher relative area increments, therefore, all regions with $\Phi>T h_{\text {areaf }}$ (eq. $11 ; R_{k}^{R}$ $k$ th reconstructed region) will be considered as noisy ones.

$$
\Phi=\frac{\sum_{R_{j} \in C_{k}} \operatorname{Area}\left(R_{j}\right)}{\text { Area }\left(R_{k}^{R}\right)}>T h_{\text {areaf }}
$$

In Fig. $2 \mathrm{c}$ and Fig. $2 \mathrm{~d}$ several adjacency maps obtained by this procedure are presented.

\section{CONCLUSIONS}

In this paper an algorithm for vessel and fibber quality characterisation is introduced. Several obstacles inherent to the type of images used in this analysis were avoided through the inclusion of a priori knowledge of some morphological properties of the intended regions. To segment these images a new contrast enhancement strategy is presented, which improves classical statistical valley search methods. Region reconstruction is performed upon constrained merge operations, with a conditional dilation operator to avoid shape distortion. To measure some of the intended characteristics an adjacency identification method is introduced. It should be noted that the described method only needs two user-defined values, which are quite intuitive.

\section{ACKNOWLEDGEMENTS}

The authors express their gratitude to Tecnocel, E.P., for the used wood samples. This project was partially supported by Praxis XXI/FCT.(CISUC).

\section{REFERENCES}

Brink, A. D. and Pendock, N. E. (1996). Minimum cross-entropy threshold selection. Pattern Recognition, 29, 179-188.

Carvalho, P., Costa, N., Ribeiro, B. and Dourado, A. (1997). Industrial visual inspection of lime balls by neural networks. 22nd Int. Conf. for Computers and Industrial Engineering, C\&IE'97, Cairo, Egypt, 64-67.

Carvalho, P. Araújo, H. and Dourado, A. (1998). Automatic structural wood quality assessment based on morphological properties. $7^{\text {th }}$ Int. Conf. On Intelligent Systems, ICIS'98, Melun, France, 181-186.

Udupta, J. K., Samarasekera, A. (1996). Fuzzy connectdness and object definition: theory, algorithms and applications in image segmentation. Graphical Models and Image Processing, 58, 246-261. 